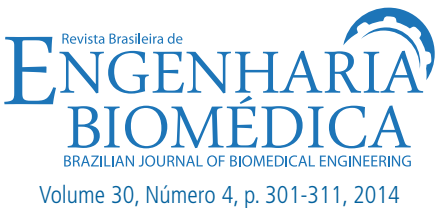

Original Article

http://dx.doi.org/10.1590/1517-3151.0543

\title{
Proposing the novelty classifier for face recognition
}

\author{
Cicero Ferreira Fernandes Costa Filho*, Thiago de Azevedo Falcão, Marly Guimarães Fernandes Costa, \\ José Raimundo Gomes Pereira
}

\begin{abstract}
Introduction: Face recognition, one of the most explored themes in biometry, is used in a wide range of applications: access control, forensic detection, surveillance and monitoring systems, and robotic and human machine interactions. In this paper, a new classifier is proposed for face recognition: the novelty classifier. Methods: The performance of a novelty classifier is compared with the performance of the nearest neighbor classifier. The ORL face image database was used. Three methods were employed for characteristic extraction: principal component analysis, bi-dimensional principal component analysis with dimension reduction in one dimension and bi-dimensional principal component analysis with dimension reduction in two directions. Results: In identification mode, the best recognition rate with the leave-one-out strategy is equal to $100 \%$. In the verification mode, the best recognition rate was also $100 \%$. For the half-half strategy, the best recognition rate in the identification mode is equal to $98.5 \%$, and in the verification mode, $88 \%$. Conclusion: For face recognition, the novelty classifier performs comparable to the best results already published in the literature, which further confirms the novelty classifier as an important pattern recognition method in biometry.
\end{abstract}

Keywords Face recognition, Novelty classifier, K Nearest Neighbor, Principal Component Analysis.

\section{Introduction}

Face recognition, one of the most explored themes in biometry, is used in a wide range of applications: access control, forensic detection, surveillance and monitoring systems, and robotic and human machine interactions; therefore, it is a technology with high commercial value. Table 1 presents a literature review in the area of face recognition, showing the following details: publication year, authors, title, pre-processing, characteristic extraction, classifier and results.

A face recognition system generally comprises the following phases: image acquisition, pre-processing, characteristic extraction and classification.

The pre-processing phase aims at making a comparison possible between images of either different individuals or of the same individual taken at different moments. The following operations are commonly used in this phase: image size adjustment, eye centralization or gray-level scale adjustment. Sahoolizadeh et al. (2008) removed background information by reducing the image size to $40 \times 40$ pixels. In the study of Shermina (2011), aiming to correct luminance non-uniformity, a luminance normalization was employed.

Some authors (Le and Bui, 2011; Noushath et al., 2006; Oliveira et al., 2011; Yang et al., 2004, 2005; Zhang and Zhou, 2005), nevertheless, do not apply any pre-processing to the face image.

The majority of algorithms for characteristic extraction used in face recognition are based on statistical methods: Principal Component Analysis (PCA) (Chan et al., 2010; Kirby and Sirovich, 1990; Perlibakas, 2004; Turk and Pentland, 1991); bidimensional PCA with dimension reduction in one direction (2DPCA) (Rouabhia and Tebbikh, 2011; Yang et al., 2004); bi-dimensional PCA with dimension reduction in two directions $\left((2 \mathrm{D})^{2} \mathrm{PCA}\right)$ (Zhang and Zhou, 2005); Linear Discriminant Analysis (LDA) (Belhumeur et al., 1997; Chan et al., 2010); bi-dimensional LDA with dimension reduction in one direction (2DLDA) (Yang et al., 2005) and bidimensional LDA with dimension reduction in two directions ((2D)²LD) (Noushath et al., 2006).

In the classification step, the following methods have been published: K Nearest Neighbor (KNN) associated with Euclidian distance (Noushath et al., 2006; Yang et al., 2004, 2005; Zhang and Zhou, 2005), Neural Networks (NN) (MageshKumar et al., 2011) and Support Vector Machines (SVM) (Le and Bui, 2011; Oliveira et al., 2011).

This paper proposes using the novelty classifier for face recognition. The performance of the novelty classifier was compared with the performance of the KNN classifier. The ORL face image database was used. The following methods were used for characteristic extractions: PCA, 2DPCA and (2D) ${ }^{2} \mathrm{PCA}$. The performance of both classifiers was evaluated in verification and identification mode. 


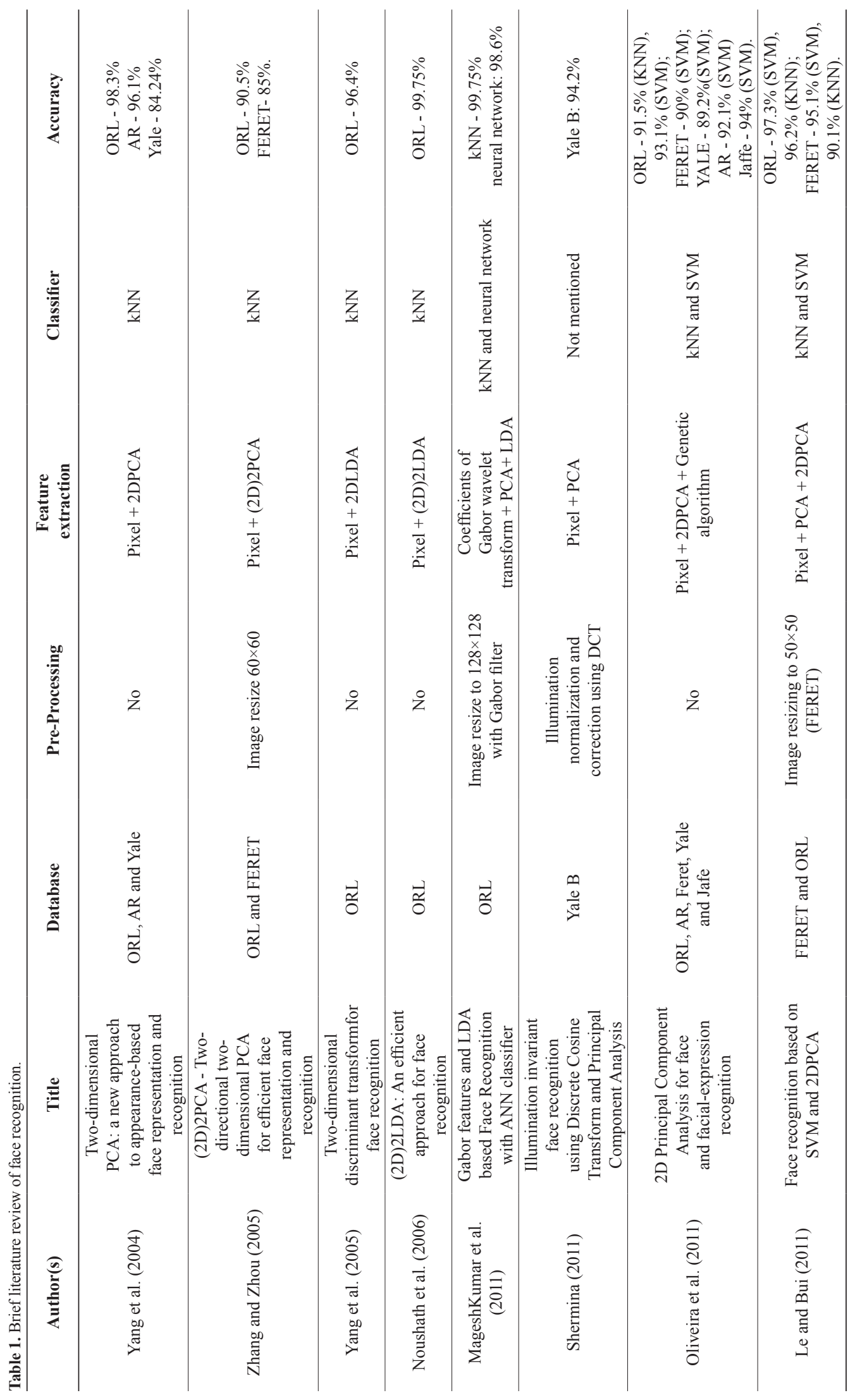


The methods section is devoted to presenting the database used, the novelty classifier, the methods used for characteristic extraction and details of how the experiments were conducted. The results section presents curves of recognition rate behavior with the number of principal components of the extraction characteristic methods. Tables with the best values of recognition rate are also shown. In the discussion section, the obtained results are compared with other results previously published in the literature and a brief discussion about novelty filter behavior is included.

\section{Methods}

\section{ORL Database}

A performance comparison between different methods of face recognition is only possible because certain institutions and research groups provide face image databases on the Internet, which allow for standardization of findings. The most used databases are Yale, Yale B, ORL, AR, FERET and JAFFE.

In this paper, the ORL database was used (AT\&T..., 2014). This database, released by Olivetti Research Laboratory, is comprised of 400 face images, each with a size of $92 \times 112$ pixels. The face images are of 40 individuals ( 36 men and 4 women) with 10 images for each individual. The ORL database was chosen because it offers a great variety of image types; the facial images of an individual were captured at different times with different conditions of illumination (originating on the right, left and center) and facial expression (normal, happy, sad, sleepy and closed eyes). All images were captured with a uniform background.

The original images of the ORL database were employed. No photometric or geometric pre-processing was performed.

\section{Nearest Neighbor Classifier (KNN)}

$\mathrm{KNN}$ is a method that classifies a sample based on $\mathrm{k}$ votes of the nearest objects in the characteristic space. If $\mathrm{k}=1$, the sample is classified as belonging to the class of the nearest neighbor and the classifier method is called the nearest neighbor classifier (Theodoridis and Koutroumbas, 2009).

In this study, the distance used was the Euclidian distance, as shown in Equation 1:

$$
d(a, b)=\sqrt{\sum_{i=1}^{m}\left(a_{i}-b_{i}\right)^{2}}
$$

Where: $a$ is the test image ( $a$ is a pixel of image a) and $b$ is an image of the training set $\left(b_{i}\right.$ is a pixel of image b). Both images are projected in the subspace generated by the method of characteristic extraction: $m<n, n=$ number of pixels in the image.

\section{Novelty classifier}

The concept of a novelty filter is used in the definition of a novelty classifier. We describe the novelty filter and next, the novelty classifier.

\section{Novelty filter concept}

A novelty filter is a type of auto-associative memory, proposed by Kohonen (1989). Its workings can be understood through the following steps: i) store familiar patterns in memory; ii) apply a given input to the memory input and retrieve the pattern that best matches the input from the memory; iii) define the novelty as the difference between the given input and the retrieved pattern.

The approach used in this paper to calculate the novelty uses the concept of auto-associative memory as an orthogonal projection. In this case, the novelty filter is submitted to a supervised training that uses the Gram-Schmidt orthogonalization method to produce a set of orthogonal vectors.

Consider a group of vectors $\left\{\mathrm{x}_{1}, \mathrm{x}_{2}, \ldots, \mathrm{x}_{\mathrm{m}}\right\} \subset \mathrm{R}^{\mathrm{n}}$ forming a base that generates a subspace $\mathrm{L} \subset \mathrm{R}^{\mathrm{n}}$, with $\mathrm{m}<\mathrm{n}$. An arbitrary vector $\mathrm{x} \in \mathrm{R}^{\mathrm{n}}$ can be decomposed in two components, $\hat{x}$ and $\widetilde{x}$, where $\hat{x}$ is a linear combination of vectors $x_{k}$; in other words, $\hat{x}$ is the orthogonal projection of $x$ on subspace $L$ and $\widetilde{x}$ is the orthogonal projection of $\mathrm{x}$ on a subspace $\mathrm{L} \perp$ (orthogonal complement of L). Figure 1 illustrates the orthogonal projections of $\mathrm{x}$ in a tridimensional space. It can be shown through the projection theorem, that $\widetilde{\mathrm{x}}$ is unique and has a minimum norm. So, $\hat{x}$ is the best representation of $x$ in subspace $L$.

The $\widetilde{x}$ component of the vector can be thought of as the result of an operation of information processing, with very interesting properties. It can be assumed that $\widetilde{\mathrm{X}}$ is the residue remaining when the best linear combination of the old patterns (vector base $x_{k}$ ) is adjusted to express vector $\mathrm{x}$. Thus, it is possible to say

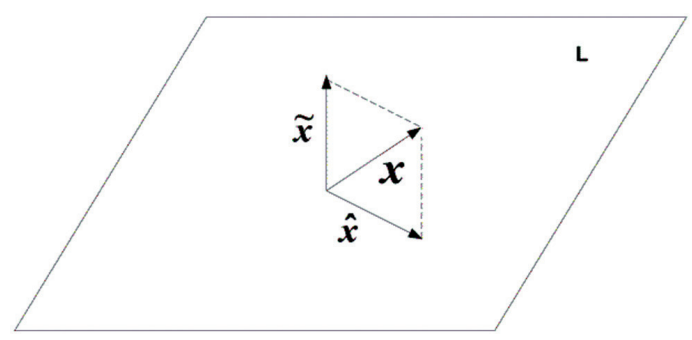

Figure 1. Illustration of a novelty filter concept using the GramSchmidt Orthogonalization method. 
that $\widetilde{x}$ is the new part of $\mathrm{x}$ that is not explained by the old patterns. This component is named novelty and the system that extracts this component from $\mathrm{x}$ can be named the novelty filter. The vector base $x_{k}$ can be understood as the memory of the system, while $\mathrm{x}$ is a key through which information is associatively searched in the memory.

It can be shown that the decomposition of an arbitrary vector $\mathrm{x} \in \mathrm{R}^{\mathrm{n}}$ in its orthogonal projections $\hat{\mathrm{x}} \in L \subset \mathrm{R}^{\mathrm{n}}$ and $\widetilde{\mathrm{x}} \in \mathrm{L} \perp$ can be obtained from a linear transformation, using a symmetric matrix $P$, so $\hat{\mathrm{x}}=\mathrm{P} . \mathrm{x}$ and $\widetilde{\mathrm{x}}=(\mathrm{I}-\mathrm{P}) . \mathrm{x}$. The matrix $P$ is named the orthogonal projector operator in $L$ ( $P$ is named the novelty filter), and ( $\mathrm{I}-\mathrm{P})$ is the orthogonal projector in subspace $\mathrm{L} \perp$.

Consider a matrix $\mathrm{X}$ with $\mathrm{x}_{1}, \mathrm{x}_{2}, \ldots, \mathrm{x}_{\mathrm{k}}$, with $\mathrm{k}<\mathrm{n}$ as its columns. Suppose that the vectors $x_{i} \in R^{n}$, $\mathrm{i}=1,2, \ldots, \mathrm{k}$ span the subspace $\mathrm{L}$. As cited above, the decomposition of $\mathrm{x}=\hat{\mathrm{x}}+\widetilde{\mathrm{x}}$ is unique and $\widetilde{\mathrm{x}}$ can be determined through the condition that it is orthogonal to all columns of $\mathrm{X}$. In other words:

$$
\left\langle\tilde{x}^{T} \cdot X\right\rangle=0
$$

The Penrose (1955) solution to Equation 2 is given by:

$$
\tilde{x}^{T}=y^{T}\left(I-X \cdot X^{+}\right)
$$

Where:

$y$ is a vector with the same dimension of $\widetilde{\mathrm{x}}$ and $\mathrm{X}^{+}$is the pseudo-inverse matrix of $X$.

Using the properties of symmetry and idempotence of the pseudo-inverse matrix, it follows that:

$$
\begin{aligned}
& \left\langle x^{T} \cdot \tilde{x}\right\rangle=x^{T} \cdot\left(I-X \cdot X^{+}\right) \\
& \left\langle x^{T} \cdot \tilde{x}\right\rangle=\left\langle\tilde{x}^{T} \cdot x\right\rangle=y^{T} \cdot\left(I-X \cdot X^{+}\right)^{2}
\end{aligned}
$$

Comparing Equations 4 and 5, it follows that $\mathrm{y}=\mathrm{x}$. So $\widetilde{\mathrm{x}}$ can be written as:

$\tilde{x}=\left(I-X \cdot X^{+}\right) \cdot x$

Because $\widetilde{\mathrm{X}}$ is unique, it follows that: $\mathrm{I}-\mathrm{P}=\mathrm{I}-\mathrm{X} \cdot \mathrm{X}^{+}$ and $\mathrm{P}=\mathrm{X} \cdot \mathrm{X}^{+}$.

When working with images, the calculation of projection matrix $P$ becomes an immense and time-consuming computational task because of the dimensions involved. Each column of matrix $X$ is a reference pattern or, in neural network terminology, a training vector. A vector such as this is constructed by stacking the image columns. For example, with images of $128 \times 128$ pixels, the dimension of the column vector is $n=16,384$ and the dimension of the $X$ matrix is $n \times N$, where $N$ is the number of training vectors (images). Thus, in this case, $P$ results in a square matrix with dimensions of 16,384 . Thus, it is preferable to obtain the novelty ${ }^{{ }^{x}}$ through an iterative technique based on the classical Gram-Schmidt orthogonalization method. This method results in the creation of a base of vectors that are mutually orthogonal, $\left\{\mathrm{h}_{1}, \mathrm{~h}_{2}, \ldots, \mathrm{h}_{\mathrm{n}}\right\} \in \mathrm{R}^{\mathrm{n}}$, from the training vectors, $\left\{\mathrm{x}_{1}, \mathrm{x}_{2}, \ldots, \mathrm{x}_{\mathrm{n}}\right\} \in \mathrm{R}^{\mathrm{n}}$.

To build a base of mutually orthogonal vectors, a direction is first chosen; for example, the direction $\mathrm{x}_{1}$, so:

$h_{1}=x_{1}$

In the sequence, this expression is used:

$h_{k}=x_{k}-\sum_{j=1}^{k-1} \frac{\left\langle x_{k}, h_{j}\right\rangle}{\left\|h_{j}\right\|^{2}} \cdot h_{j}$, for $k=2,3, \ldots m$

Where:

$\left\langle x_{\mathrm{k}}, h_{\mathrm{j}}\right\rangle$ is the inner product of $x_{\mathrm{k}}$ and $h_{\mathrm{j}}$.

The way that vectors $h_{\mathrm{j}}$ are constructed, it follows that the set $\left\{\mathrm{h}_{1}, \mathrm{~h}_{2}, \ldots, \mathrm{h}_{\mathrm{n}}\right\}$ spans the same subspace as the set $\left\{\mathrm{x}_{1}, \mathrm{x}_{2}, \ldots, \mathrm{x}_{\mathrm{n}}\right\}$.

Given a sample $x$, to obtain the novelty $\widetilde{\mathrm{x}}$, it is necessary to continue the process described by Equation 7 one step more: $\widetilde{\mathrm{x}}=h_{\mathrm{n}+1}$.

\section{Binary and multiclass classifiers using novelty} filters

Differing from neural networks, the training set of a novelty filter consists only of sample vectors that belong to a given class.

Suppose that the training set consists of the vectors set, $\left\{\mathrm{x}_{1}, \mathrm{x}_{2}, \mathrm{x}_{3}, \ldots, \mathrm{x}_{\mathrm{n}}\right\}$, belonging to a given class. The training step consists in obtaining the set, $\left\{\mathrm{h}_{1}, \mathrm{~h}_{2}, \mathrm{~h}_{3}, \ldots, \mathrm{h}_{\mathrm{n}}\right\}$, according to Equation 8 . It should be noted that, before submitting the input vectors to the Gram-Schmidt orthogonalization method, they should be normalized. Figure 2a shows the block diagram of a unary classifier training using the novelty filter.

Figure $2 \mathrm{~b}$ shows a block diagram of a unary classifier using a novelty filter. For classifying a sample, the Gram-Schmidt orthogonalization process is run as shown according to Equation 8, generating a novelty vector $\widetilde{x}_{1}$. In the sequence, the novelty vector norm is extracted and compared to a decision threshold value.

Figure 2c shows a block diagram of a multiclass classifier using novelty filters. For classifying a sample, the Gram-Schmidt orthogonalization process is run as shown for each one of the classifiers $C_{i}$, according to Equation 8, thus generating a set of 


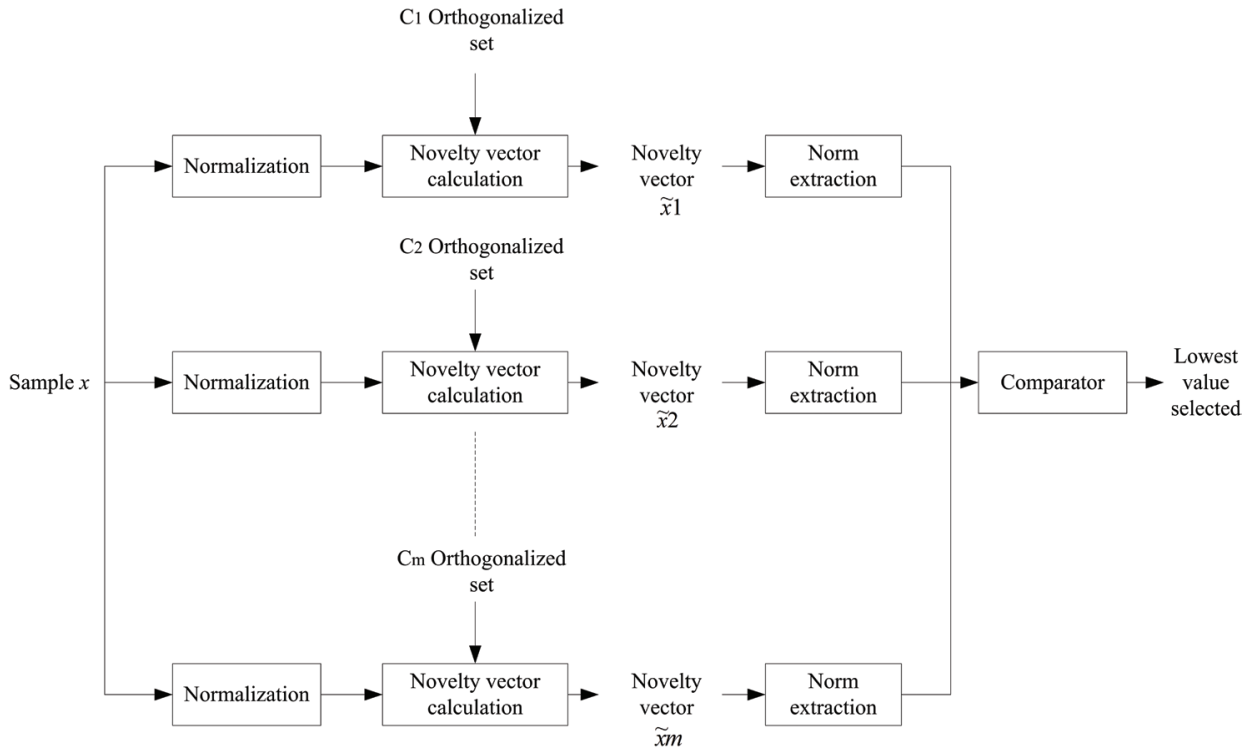

Figure 2. (a) Training the novelty filter; (b) Binary classifier using the novelty filter; (c) Multiclass classifier using novelty filters.

novelty vectors $\left\{\sim \mathrm{x}_{1}, \mathrm{x}_{2}, \ldots, \sim \mathrm{x}_{\mathrm{n}}\right\}$, one for each classifier $C_{\mathrm{i}}$. In the sequence, the novelty vectors norms are extracted and their magnitudes are compared. The one with the lowest value defines the class to which the sample belongs. This multiclass classifier requires training $m$ classifiers and uses all of them in the classification task.

The authors used the novelty classifier concept in some previous works (Costa and Moura, 1995; Costa Filho et al., 2013; Melo et al., 2014). In the first, the novelty classifier is applied to natural gas leak detection. In the second, the novelty classifier is applied to iris recognition and in the last one, the novelty classifier is applied to cancer diagnostics in scintmammographic images.

\section{Characteristic extraction}

As stated earlier, three methods were used for characteristic extraction of face images from the ORL database: PCA, 2DPCA and (2D) ${ }^{2}$ PCA.

PCA is defined as the task of finding a sub-space in such a way that the variance of the orthogonal data projections in it is maximized (Hotelling, 1993). This sub-space is called the principal sub-space. Consider $\mathrm{X}=\left\{\mathrm{x}_{1}, \mathrm{x}_{2}, \ldots, \mathrm{x}_{\mathrm{N}}\right\}$ a set of original data and $S$ the covariance matrix given by Equation 9:
$S=\frac{1}{N} \sum_{n=1}^{N}\left(x_{n}-\bar{x}\right) \cdot\left(x_{n}-\bar{x}\right)^{T}$

To maximize the criteria given in the preceding paragraph, the projection of a vector $x_{i}$ in the principal subspace is given by Equation 10:

$x_{p i}=U^{T} \cdot x_{i}$

Matrix $U$ if given by Equation 11:

$U=\left[u_{1}, u_{2}, \ldots, u_{m}\right]$

Where: $u_{1}, u_{2}, \ldots, u_{\mathrm{m}}$ are auto-vectors that correspond to the highest auto-values of covariance matrix S. If the original vector $x_{\mathrm{i}}$ is $d x 1$, the dimension of the projected vector is $m x 1$. Each one of the $m$ vectors is called a principal component. The dimensional reduction occurs because $m<d$. The lower the number of auto-vectors in matrix $U$, the higher the dimensional reduction.

To apply the PCA method with images, it is necessary that a $2 \mathrm{D}$ image be converted into a $1 \mathrm{D}$ vector. Therefore, the vector dimension is very high, which generates a high dimensional covariance matrix $S$, rendering it difficult to find its auto-vectors.

The 2DPCA method, proposed by Yang et al. (2004) solves this problem. In this technique, the covariance matrix is given by Equation 12: 


$$
G_{H}=\frac{1}{N} \sum_{i=1}^{N}\left(A_{i}-\bar{A}\right)^{T} \cdot\left(A_{i}-\bar{A}\right)
$$

Where:

$$
\begin{aligned}
& A_{\mathrm{i}} \rightarrow \text { ith image of a set with } \mathrm{N} \text { mxn images } \\
& \bar{A} \rightarrow \text { average image } \\
& G_{H} \rightarrow(n x m)(m x n) \rightarrow n x n
\end{aligned}
$$

Matrix $U$ projects the original image aiming to maximize the projected covariance image, according to Equation 13:

$$
A_{\mathrm{pi}}=A_{\mathrm{i}} \cdot U
$$

Matrix $U$ if given by Equation 14:

$$
U=\left[u_{1}, u_{2}, \ldots, u_{\mathrm{q}}\right]
$$

Where: $u_{1}, u_{2}, \ldots, u_{\mathrm{q}}$ are auto-vectors that correspond to the highest auto-values of covariance matrix $G_{\mathrm{H}}$. If the original matrix $A$ is $m x n$, the dimension of the projected matrix $A_{\mathrm{pi}}$ is $m x q$. The dimensional reduction occurs because $q<n$. The lower the number of auto-vectors in matrix $U$, the higher the dimensional reduction.

The 2D ${ }^{2} \mathrm{PCA}$ method, proposed by Zhang e Zhou (2005), reduces the original image dimension in both horizontal and vertical directions. This method consists of finding two projection matrices, as shown in Equation 15:

$U=\left[u_{1}, u_{2}, \ldots, u_{\mathrm{q}}\right]$ e $V=\left[v_{1}, v_{2}, \ldots, v_{\mathrm{d}}\right]$

Matrix $U$ is used to reduce dimensions in the horizontal direction and is the same one determined in the 2DPCA method. Matrix V is used to reduce dimensions in the vertical direction and is given in Equation 16:

$$
G_{V}=\frac{1}{N} \sum_{i=1}^{N}\left(A_{i}-\bar{A}\right)\left(A_{i}-\bar{A}\right)^{T}
$$

Matrix V projects the original image and aims to maximize the projected covariance image, according to Equation 17:

$A_{\mathrm{pi}}=V^{\mathrm{T}} A_{\mathrm{i}}$

Matrix $V$ if given by Equation 18:

$V=\left[v_{1}, v_{2}, \ldots, v_{\mathrm{r}}\right]$

Where: $\mathrm{v}, v_{2}, \ldots, v_{\mathrm{r}}$ are auto-vectors that correspond to the highest auto-values of covariance matrix $G_{\mathrm{V}}$. If the original matrix $A$ is $m x n$, the dimension of the projected matrix $A_{\mathrm{pi}}$ is $r x m$. The dimensional reduction occurs because $r<m$. The lower the number of auto-vectors in matrix $U$, the higher the dimensional reduction. The final projected image is given by Equation 19:

$A_{\text {fpi }}=V^{\mathrm{T}} A_{\mathrm{i}} U$
The dimension of $A_{\text {fpi }}$ is rxq.

In this work, for testing the novelty classifier and the KNN classifier, the number of principal components of each one of these methods was varied. For the PCA method, the number of components varied between 0 and 360, in steps of 1. For the 2DPCA method, the matrix components varied between $112 \times 2$ and $112 \times 20$, in steps of $\times 1$. For the $(2 \mathrm{D})^{2} \mathrm{PCA}$ method, the matrix components varied between $5 \times 5$ and $30 \times 30$, in steps of $5 \times 5$.

Because the novelty classifier uses vectors as inputs, the matrices resulting from the 2DPCA and (2D) ${ }^{2}$ PCA methods were converted into vectors.

\section{Experiments}

To compare the performance of the multiclass novelty classifier with the performance of the KNN classifier, two training-test strategies were used: half-half and leave-one-out (Sonka and Fitzpatrick, 2000).

The experiments were performed both in identification and verification modes. In identification mode, a biometric sample is compared with the models of individuals previously registered in the biometric database. The system can provide two answers: a list of $\mathrm{k}$ individuals with the most similarities to the sample or an indication that the sample is not registered in the biometric database. If the list contains only one individual, then the recognition is said to be rank-1. If the list contains $\mathrm{k}$ individuals, the recognition is said to be rank-k.

In verification mode, an individual communicates a particular identity to the biometric system. Verification consists of comparing this identity with the same one previously registered in the biometric database. If, according to a given criteria, the comparison results are positive, the individual identity is accepted as true and the individual is considered genuine. Otherwise, the individual identity is not accepted as true and the individual is considered an impostor.

The following recognition rate was used to evaluate classifiers (Jain et al., 2011):

$$
\text { Recognition Rate }=\frac{\text { Number of recognized faces }}{\text { Number of testing faces }}
$$

For identification mode, curves were obtained showing the behavior of the rank-1 recognition rate versus the number of principal components of the characteristic extraction methods.

For verification mode, curves were obtained showing the behavior of the recognition rate versus the number of principal components of the characteristic extraction methods, using a false acceptance rate (FAR) of $0.1 \%$. FAR is defined as the probability of classifying an impostor as genuine. The False Rejection 
Rate (FRR) is defined as the probability of classifying a genuine as an impostor. A smaller FAR indicates a lower probability of an impostor being accepted as genuine. Biometric systems prefer a lower FAR. In verification mode, the equivalent error rate was also calculated and was defined as the intersection point of the two probability distributions of FAR and FRR.

For both modes, we presented the best results for the recognition rate of half-half and leave-one-out training-test strategies (Sonka and Fitzpatrick, 2000).

In the half-half strategy, the novelty filter training set of each individual was comprised of half of the images (5 images). The other half was used for testing. The testing set for each individual was comprised of genuine and impostor images, 5 and 195 images, respectively. There was no superposition between training and test sets. The experiment was repeated 10 times with different training and testing sets.

In the leave-one-out strategy, the novelty filter training set of each individual was comprised of 9 images. The tenth image was used for testing. The testing set was comprised of genuine and impostor images, 1 and 39 images, respectively. As there were ten images per individual, the training and testing were repeated 10 times.

These strategies are the most commonly used in the literature. Their choice allows a comparison of the results obtained in this work with results of other previously published work.

\section{Results}

Figure 3 shows, for both classifiers, curves of rank1 recognition rates versus number of principal components for identification mode using the three methods of characteristic extraction: PCA, 2DPCA and $(2 \mathrm{D})^{2} \mathrm{PCA}$.

In the curves showing PCA results, the horizontal axis dimension, corresponds to the $\mathrm{m}$ dimension (number of principal components) of the matrix components given in Equation 11. In the curves showing 2DPCA results, the horizontal axis corresponds to the $q$ dimension (number of principal components) of the matrix components given in Equation 14. In the curves showing $2 \mathrm{D}^{2} \mathrm{PCA}$ results, the horizontal axis corresponds to the $q$ or $r$ dimension because $q=r$ (number of principal components) of the matrix components given in Equation 19.

Table 2 shows the best performance of both classifiers in identification mode. As noted, both classifiers present a higher rank-1 recognition rate with the leave-one-out training-test strategy.

Figure 4 shows, for both classifiers, recognition rate versus number of principal components for verification mode using the three methods of characteristic extraction: PCA, 2DPCA and (2D) ${ }^{2}$ PCA.

Table 3 shows the best performance of both classifiers in verification mode, with $\mathrm{FAR}=0.1 \%$.

Concerning the time each classifier takes to classify a sample, we observed that the KNN classifier is faster than the novelty classifier. For classifying 200 samples, using the 2DPCA method, the KNN classifier with a $112 \times 5$-matrix feature, takes approximately 1648 milliseconds, while a novelty classifier takes approximately 1765 milliseconds. This test was made using a computer with an $15-2540 \mathrm{M} 2.6 \mathrm{GHz}$ Processor, running Matlab 2012.

\section{Discussion}

The results of Figure 3 showed that, for PCA and (2D)2PCA, the recognition rate for identification mode stabilizes with a lower number of principal components, 10 and $25(5 \times 5)$, respectively. For 2DPCA, however, the recognition rate has unstable behavior with increasing principal components. As shown in Figure 4, similar behavior is observed for verification mode. In both modes, using the same number of principal components, the performance of the novelty classifier is better than the performance of the KNN classifier.

The results in Tables 2 and 3 show that, for both identification and verification modes, the best recognition rate of the novelty classifier occurs with the leave-one-out strategy and, similar to what was observed in the last paragraph, the performance of the novelty classifier is better than the performance of the KNN classifier. In identification mode, the recognition rate with the leave-one-out strategy is equal to $100 \%$ with PCA, 2DPCA and (2D) ${ }^{2}$ PCA. These results were obtained with the corresponding principal components matrices: PCA $-25 \times 1$, 2 DPCA $-112 \times 2$ and $2 D^{2} P C A-5 \times 5$. In verification mode, the recognition rate is $100 \%$ with PCA and 2DPCA and $97.5 \%$ with $(2 \mathrm{D})^{2} \mathrm{PCA}$. These results were obtained with the corresponding principal components matrices: PCA - 144×1, 2DPCA - 112×8 and $2 \mathrm{D}^{2} \mathrm{PCA}-10 \times 10$. For the half-half strategy, the best recognition rate in the identification mode was obtained with (2D) ${ }^{2} \mathrm{PCA}(98.5 \%)$, and, in the verification mode, with PCA $(88 \%)$.

In the literature, using the ORL database and the 2DPCA method, Yang et al. (2004) achieved the best results with a principal component matrix of $112 \times 3$. The second dimension of this matrix is between 3 and 8 , determined in this work using 2DPCA method, for the identification and verification modes, respectively. Zhang and Zhou (2005), using the same database and $2 \mathrm{D}^{2} \mathrm{PCA}$, achieved the best results with a principal 
component matrix of $27 \times 26$. The dimensions of this last matrix are very different from the dimensions $5 \times 5$ and $10 \times 10$, which were determined in this work using the $2 \mathrm{D}^{2} \mathrm{PCA}$ method for identification and verification modes, respectively.

(a)

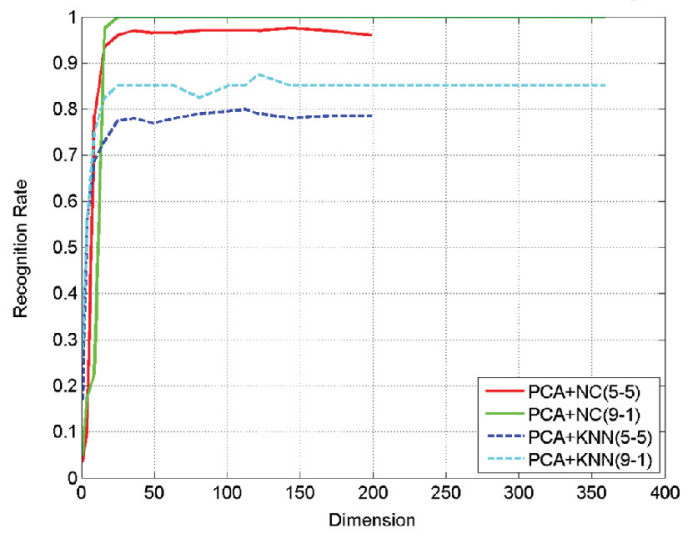

Comparing the results of this work with previously published results using the ORL database and shown previously (Table 1), we observed that the novelty classifier shows results comparable with the best results published in the literature

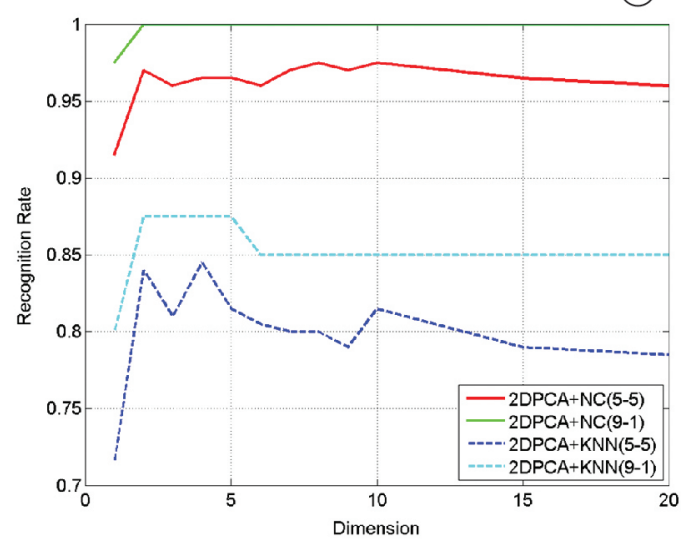

(c)

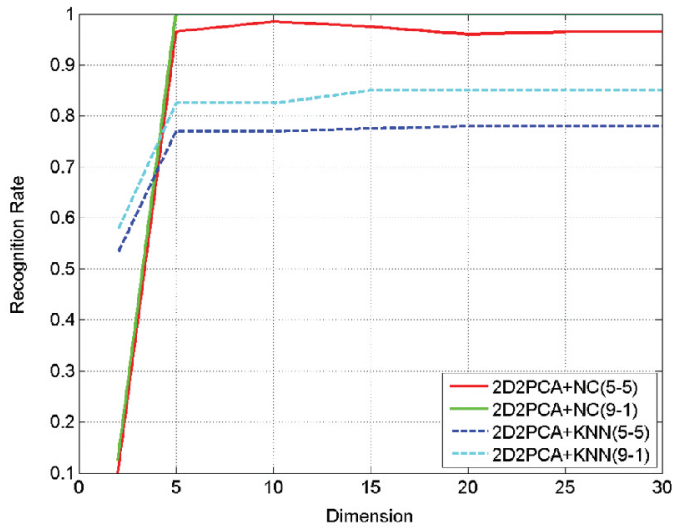

Figure 3. Rank-1 recognition rate versus number of principal components. (a) PCA; (b) 2DPCA; (c) 2D²PCA. NC - Novelty Classifier; 5-5 - half-half training-test strategy; 9-1 - leave-one-out training-test strategy.

Table 2. Rank-1 best recognition rate of both classifiers in identification mode.

\begin{tabular}{|c|c|c|c|c|}
\hline Classifier & $\begin{array}{c}\text { Training-Test } \\
\text { Strategy }\end{array}$ & $\begin{array}{c}\text { Feature } \\
\text { Extraction Method }\end{array}$ & $\begin{array}{c}\text { Number of Principal } \\
\text { Components } \\
\text { (Dimension) }\end{array}$ & Value \\
\hline \multirow{6}{*}{ Novelty Classifier } & \multirow{3}{*}{ half-half } & PCA & 144 & $97.5 \%$ \\
\hline & & 2DPCA & $112 \times 8$ & $97.5 \%$ \\
\hline & & $(2 \mathrm{D})^{2} \mathrm{PCA}$ & $10 \times 10$ & $98.5 \%$ \\
\hline & \multirow{3}{*}{ leave-one-out } & PCA & 25 & $100 \%$ \\
\hline & & 2DPCA & $112 \times 2$ & $100 \%$ \\
\hline & & $(2 \mathrm{D})^{2} \mathrm{PCA}$ & $5 \times 5$ & $100 \%$ \\
\hline \multirow{6}{*}{ K Nearest Neighbor } & \multirow{3}{*}{ half-half } & PCA & 112 & $80 \%$ \\
\hline & & 2DPCA & $112 \times 4$ & $84.5 \%$ \\
\hline & & $(2 \mathrm{D})^{2} \mathrm{PCA}$ & $20 \times 20$ & $78 \%$ \\
\hline & \multirow{3}{*}{ leave-one-out } & PCA & 121 & $87.5 \%$ \\
\hline & & 2DPCA & $112 \times 2$ & $87.5 \%$ \\
\hline & & $(2 \mathrm{D})^{2} \mathrm{PCA}$ & $15 \times 15$ & $85 \%$ \\
\hline
\end{tabular}


with both the leave-one-out strategy and the halfhalf strategy. The results obtained with the KNN classifier in this work, however, were worse than those obtained with the novelty classifier and worse than others previously obtained in the literature, as shown in Table 1.

We would like to emphasize a positive characteristic of the novelty classifier, which is its (a)

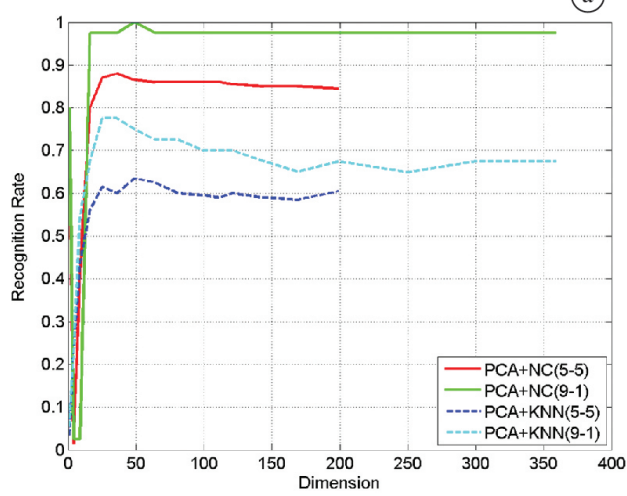

(b)

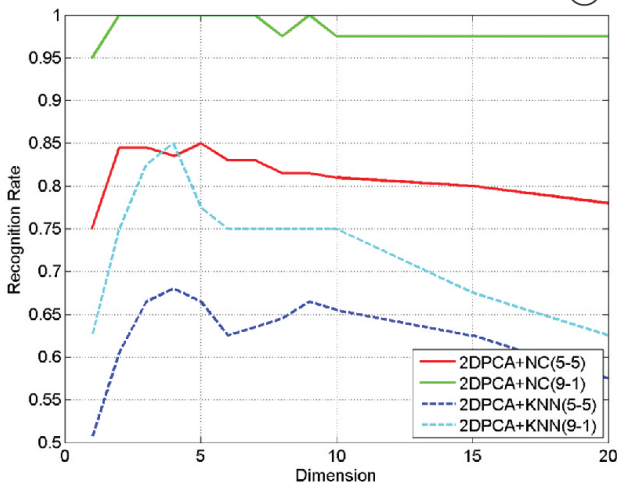

(c)

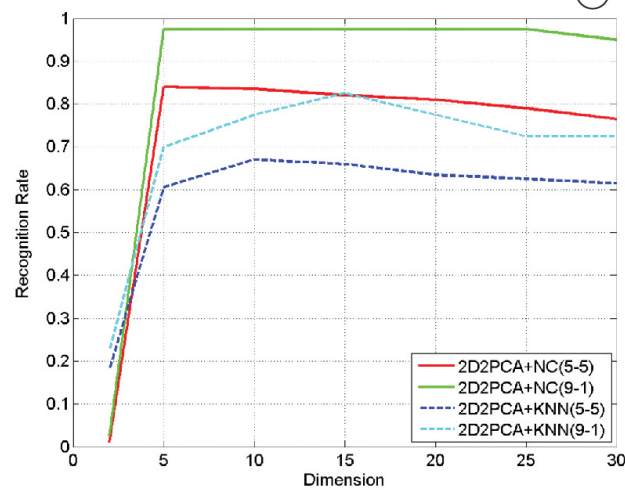

Figure 4. Recognition rate versus number of principal components for verification mode. (a) PCA; (b) 2DPCA; (c) (2D)2PCA. NC - Novelty Classifier; 5-5 - half-half training-test strategy; 9-1 - leave-one-out training-test strategy.

Table 3. Best recognition rate of both classifiers in verification mode with $\mathrm{FAR}=0.1 \%$.

\begin{tabular}{|c|c|c|c|c|c|c|}
\hline \multirow[b]{2}{*}{ Classifier } & \multirow[b]{2}{*}{$\begin{array}{c}\text { Training-Test } \\
\text { Strategy }\end{array}$} & \multirow[b]{2}{*}{$\begin{array}{c}\text { Feature } \\
\text { Extraction } \\
\text { Method }\end{array}$} & \multicolumn{2}{|c|}{ Recognition Rate } & \multicolumn{2}{|c|}{ Equal Error Rate } \\
\hline & & & $\begin{array}{c}\text { Number of } \\
\text { Principal } \\
\text { Components } \\
\text { (Dimension) }\end{array}$ & Value & $\begin{array}{c}\text { Number of } \\
\text { Principal } \\
\text { Components } \\
\text { (Dimension) }\end{array}$ & Value \\
\hline \multirow{6}{*}{$\begin{array}{l}\text { Novelty } \\
\text { Classifier }\end{array}$} & \multirow{3}{*}{ half-half } & PCA & 36 & $88 \%$ & 49 & $3.08 \%$ \\
\hline & & 2DPCA & $112 \times 5$ & $85 \%$ & $112 \times 4$ & $3.0 \%$ \\
\hline & & $(2 \mathrm{D})^{2} \mathrm{PCA}$ & $5 \times 5$ & $84 \%$ & $15 \times 15$ & $3.49 \%$ \\
\hline & \multirow{3}{*}{ leave-one-out } & PCA & 25 & $100 \%$ & 49 & $0.03 \%$ \\
\hline & & 2DPCA & $112 \times 2$ & $100 \%$ & $112 \times 2$ & $0.00 \%$ \\
\hline & & $(2 \mathrm{D})^{2} \mathrm{PCA}$ & $5 \times 5$ & $97.5 \%$ & $5 \times 5$ & $0,06 \%$ \\
\hline \multirow{6}{*}{$\begin{array}{l}\text { K Nearest } \\
\text { Neighbor }\end{array}$} & \multirow{3}{*}{ half-half } & PCA & 49 & $63.5 \%$ & 25 & $5.1 \%$ \\
\hline & & 2DPCA & $112 \times 4$ & $68 \%$ & $112 \times 2$ & $5.88 \%$ \\
\hline & & $(2 \mathrm{D})^{2} \mathrm{PCA}$ & $10 \times 10$ & $67 \%$ & $10 \times 10$ & $6.52 \%$ \\
\hline & \multirow{3}{*}{ leave-one-out } & PCA & 49 & $77.5 \%$ & 49 & $3.08 \%$ \\
\hline & & 2DPCA & $112 \times 4$ & $85 \%$ & $112 \times 3$ & $3.0 \%$ \\
\hline & & $(2 \mathrm{D})^{2} \mathrm{PCA}$ & $15 \times 15$ & $82.5 \%$ & $5 \times 5$ & $2.92 \%$ \\
\hline
\end{tabular}


excellent generalization capability, even with a low number of samples in the training set. In a previous work (Costa Filho et al., 2013), when the novelty classifier was used for iris recognition, the training sets consisted of 3 or 4 iris images. In this work, with the half-half strategy, the training set consists of 5 images. In both, excellent classification rates were obtained, showing a robust generalization capability.

Although the recognition rates obtained with the novelty classifier in this work are higher, some errors occur. Figure 5 shows an error that occurs in identification mode. Figure 5a shows five images of the novelty filter base of individual A. Figure $5 \mathrm{~b}$ shows five images of the novelty filter base of individual B. Figure $5 \mathrm{c}$ shows an image sample of individual A presented to the novelty classifier. This sample image was recognized by the novelty classifier as belonging to individual $\mathrm{B}$ and not to individual A. The novelty value of this sample related to the novelty filters of individual A and B was 2111.82 and 2024.23, respectively. Observing the images of both bases and the sample image, there is no reason for this erroneous recognition. A more detailed study must be conducted for a deeper understanding of the novelty filter classifier behavior.

Future work will address the use of other extraction characteristic techniques as we apply the novelty classifier to face recognition in other face databases cited in this work.

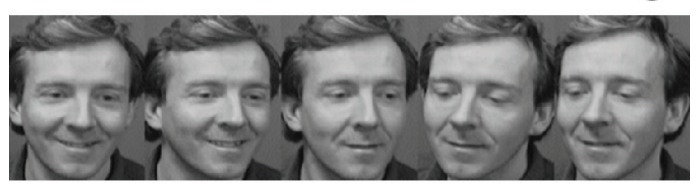

(b)

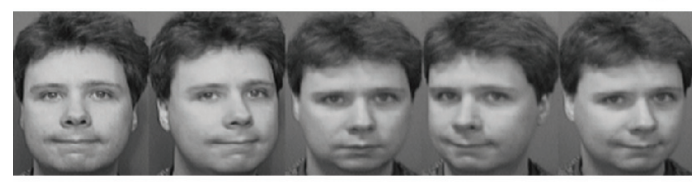

(c)

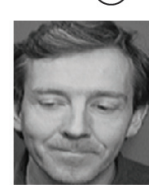

Figure 5. (a) Five images of novelty filter base of individual A; (b) Five images of novelty filter base of individual B; (c) Sample image of individual A, which was recognized by the novelty classifier as belonging to individual $\mathrm{B}$.

\section{References}

AT\&T Laboratories Cambridge. The ORL Database of Faces [internet]. Cambridge: AT\&T Laboratories Cambridge; 2014. [cited 2014 Feb 18]. Available from: http://www.cl.cam. ac.uk/research/dtg/attarchive/facedatabase.html.

Belhumeur P, Hespanha J, Kriengman D. Eigenfaces vs. fisherfaces: recognition using class specific linear projection. IEEE Transaction on Pattern Analysis and Machine Intelligence. 1997; 19(7):711-20. http://dx.doi. org/10.1109/34.598228

Chan L, Salleh SH, Ting CM. Face biometrics based on principal component analysis and linear discriminant analysis. Journal of Computer Science. 2010; 6(7):693-9. http://dx.doi.org/10.3844/jcssp.2010.693.699

Costa MGF, Moura L. Automatic assessment of scintmammographic images using a novelty filter. Proceedings of the 19th Annual Symposium on Computer Applications in Medical Care. 1995:537-41. PMid:8563342 PMCid:PMC2579151

Costa Filho CFF, Pinheiro CFM, Costa MGF, Pereira WCA. Applying a novelty filter as a matching criterion to iris recognition for binary and real-valued feature vectors. Signal, Image and Video Processing. 2013; 7(2):287-96. http://dx.doi.org/10.1007/s11760-011-0237-5

Hotelling, H. Analysis of complex statistical variables into principal components. Journal of Educational Psychology, 1993; 24 (6): 417-41.

Jain AK, Ross AA, Nandakumar K. Introduction to biometrics. New York: Springer-Verlag; 2011. http://dx.doi. org/10.1007/978-0-387-77326-1

Kirby M, Sirovich L. Application of the KL procedure for the characterization of human faces. IEEE Transactions on Pattern Analysis and Machine Intelligence. 1990; 12(1):1038. http://dx.doi.org/10.1109/34.41390

Kohonen T. Self-organization and associative memory. New York: Springer-Verlag; 1989. PMid:2562047. http:// dx.doi.org/10.1007/978-3-642-88163-3

Le TH, Bui L. Face recognition based on SVM and 2DPCA. International Journal of Signal Processing, Image Processing and Pattern Recognition. 2011; 4(3):85-94.

MageshKumar C, Thiyagarajan R, Natarajan SP, Arulselvi S, Sainarayanan G. Gabor features and LDA based face recognition with ANN classifier. International Conference on Emerging Trends in Electrical and Computer Technology (ICETECT). 2011:831-6.

Melo RO, Costa MGF, Costa Filho CFF. Using digital image processing and a novelty classifier for detecting natural gas leaks. In: Chen L, Kapoor S, Bhatia R, editors. Intelligent systems for science and information: extended and selected results from the Science and Information Conference 2013. New York: Springer International Publishing; 2014. p. 40922. (Studies in computational intelligence, 542).

Noushath S, Kumat, GH, Shivakumara P. (2D)²LDA: An efficient approach for face recognition. Pattern Recognition. 2006; 39:1396-400. http://dx.doi.org/10.1016/j. patcog.2006.01.018 
Oliveira L, Mansano M, Koerich A, Britto, AS Jr. 2D principal component analysis for face and facial-expression recognition. Computing in Science and Engineering. 2011; 13(3):9-13. http://dx.doi.org/10.1109/MCSE.2010.149

Penrose R. A generalized inverse for matrices. Proceedings of the Cambridge Philosophy Society. 1955; 51:406-13.

Perlibakas V. Distance measures for PCA-based face recognition. Pattern Recognition Letters. 2004; 25(6):71124. http://dx.doi.org/10.1016/j.patrec.2004.01.011

Rouabhia C, Tebbikh H. Efficient face recognition based on weighted matrix distance metrics and 2DPCA algorithm. Archives of Control Sciences. 2011; 21(2):207-21. http:// dx.doi.org/10.2478/v10170-010-0040-5

Sahoolizadeh AH, Heidari BZ, Dehghani CH. A new face recognition method using $\mathrm{PCA}, \mathrm{LDA}$ and neural network. World Academy of Science, Engineering and Technology. 2008; 17:7-12.

Shermina J. Illumination invariant face recognition using discrete cosine transform and principal component analysis. International Conference on Emerging Trends in Electrical and Computer Technology (ICETECT). 2011:826-30.
Sonka M, Fitzpratick JM. Handbook of medical imaging. Volume 2: Medical Imaging Processing and Analysis. Washington: Spie Press; 2000.

Theodoridis S, Koutroumbas K. Pattern recognition. 4th ed. Oxford: Elsevier Academic Press; 2009.

Turk M, Pentland A. Eigenfaces for recognition. Journal of Cognitive Neuroscience. 1991; 3(1):71-86. PMid:23964806. http://dx.doi.org/10.1162/jocn.1991.3.1.71

Yang J, Zhang D, Frangi AF, Yang J-Y. Two-dimensional PCA: a new approach to appearance-based face representation and recognition. IEEE Transactions on Pattern Analysis and Machine Intelligence. 2004; 26(1):131-7. PMid:15382693. http://dx.doi.org/10.1109/TPAMI.2004.1261097

Yang J, Zhang D, Yong X, Yang J-Y. Two-dimensional discriminant transform for face recognition. Pattern Recognition. 2005; 38(7):1125-9. http://dx.doi.org/10.1016/j. patcog.2004.11.019

Zhang D, Zhou Z-H. (2D)²PCA: Two-directional twodimensional PCA for efficient face representation and recognition. Neurocomputing. 2005; 69:224-31. http:// dx.doi.org/10.1016/j.neucom.2005.06.004

\footnotetext{
Authors

Cicero Ferreira Fernandes Costa Filho*, Marly Guimarães Fernandes Costa

Centro de Tecnologia Eletrônica e da Informação - CETELI, Universidade Federal do Amazonas - UFAM, Av. General Rodrigo Otávio Jordão Ramos, 3000, Aleixo, Campus Universitário, Setor Norte, Pavilhão Ceteli, CEP 69077-000, Manaus, AM, Brasil.
}

Thiago de Azevedo Falcão

Instituto Nokia de Tecnologia, Manaus, AM, Brasil. 